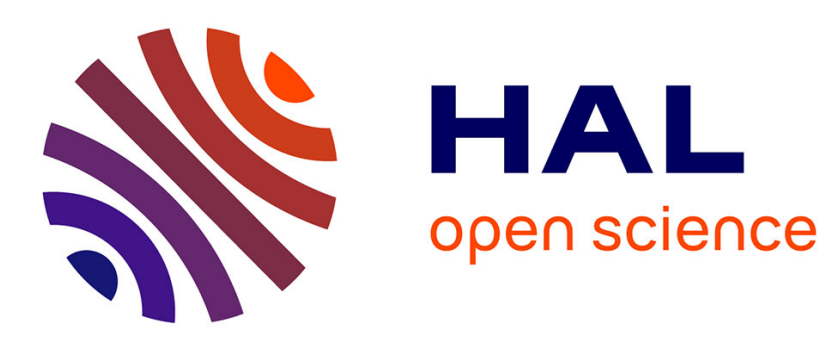

\title{
Adaptive control of microrobot in pulsatile flow
}

Lounis Sadelli, Matthieu Fruchard, Antoine Ferreira

\section{To cite this version:}

Lounis Sadelli, Matthieu Fruchard, Antoine Ferreira. Adaptive control of microrobot in pulsatile flow. IEEE Multi-Conference on Systems and Control, Oct 2014, Nice, France. hal-01071286

\section{HAL Id: hal-01071286 \\ https://hal.science/hal-01071286}

Submitted on 13 Oct 2014

HAL is a multi-disciplinary open access archive for the deposit and dissemination of scientific research documents, whether they are published or not. The documents may come from teaching and research institutions in France or abroad, or from public or private research centers.
L'archive ouverte pluridisciplinaire HAL, est destinée au dépôt et à la diffusion de documents scientifiques de niveau recherche, publiés ou non, émanant des établissements d'enseignement et de recherche français ou étrangers, des laboratoires publics ou privés. 


\title{
Adaptive control of microrobot in pulsatile flow
}

\author{
Lounis Sadelli, Matthieu Fruchard, Antoine Ferreira
}

\begin{abstract}
-
We propose an observer-based adaptive controller for a magnetic microrobot navigating in blood vessels. The pulsatile blood flow nonlinearly affects the drag force which dominates the microrobot dynamics. Instead of using a priori knowledge of the blood flow, we synthesize a mean value theorem (MVT) based observer to on-line estimate the pulsatile blood velocity. We then prove the stability of the observer-based adaptive controller in presence of parametric uncertainties. Simulation results illustrate the efficiency of the proposed approach.
\end{abstract}

\section{INTRODUCTION}

Therapeutic untethered nano and microrobots control have been an ongoing interest for many researchers since such systems can perform complex surgical procedures and reach remote places with lessened medical side effects [1], [2], [3]. Different propulsion strategies have been proposed, mainly based on magnetic deported actuation: robots with elastic flagellum [4], [5], [6], with helical flagella [7], [8], bead pulled robots or swarm of robots [9], [10], [11].

Whatever the proposed design, these systems face nonlinear forces: blood drag, electrostatic force, etc [12], [13]. Among these forces, the nonlinear drag force both prevails at a small scale and is the most disturbed by external time-varying perturbations because of the pulsatile blood flow. Its measurement, by means of either ultrasonic sensors or Magnetic Resonance Imaging (MRI) devices [14], raises two issues: the need for an end-effector servoing to track the robot, and a resolution that can not discriminate the spatial parabolic blood flow profile (see Fig 1) the robot faces depending on its position in the vessel. A priori knowledge of the blood velocity suffers from the need for an accurate knowledge of the vessel geometry in the case of a Navier Stokes computational approach or analytical approach [12] as well. Since blood velocity is required for control purposes, disturbance rejection is not appropriate either. To circumvent these issues, we have consequently proposed high gain and MVT based observers [15] respectively in [16], [17] to estimate the blood velocity and use it in the control law. Yet the robot modeling involves many physical and physiological parameters whose variability induces parametric uncertainties. Significant errors on the parameters can degrade or even lead to instability of both

L. Sadelli and M. Fruchard are with the Laboratory PRISME EA 4229, Univ. Orléans, 63 Av de Lattre de Tassigny, 18020 Bourges Cedex, France, lounis.sadelli@univ-orleans.fr matthieu.fruchardeuniv-orleans.fr

A. Ferreira is with the Laboratory PRISME EA 4229, INSA-CVL, 88 Bd Lahitolle, 18000, Bourges, France. antoine.ferreiradinsa-cvl.fr

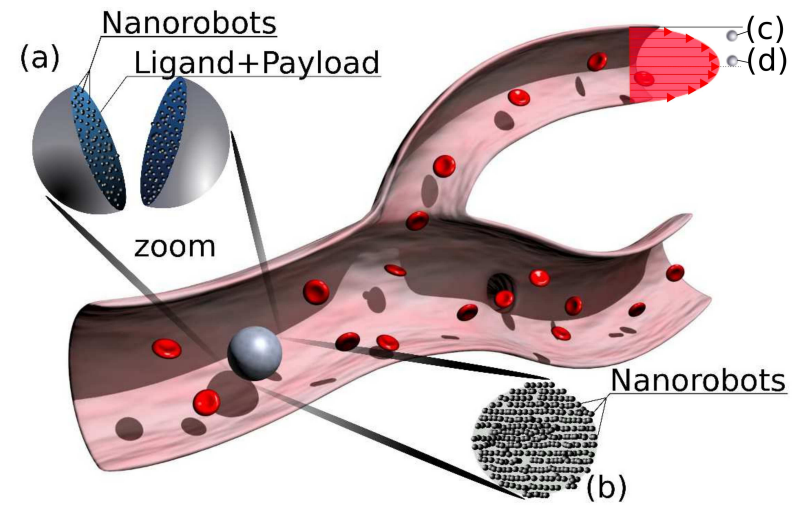

Fig. 1. Microrobot in a blood vessel. (a) binded by ligand, including a payload; (b) binded by magnetic and interaction forces. The microrobots (c) and (d) do not face the same drag force because of the parabolic blood flow profile.

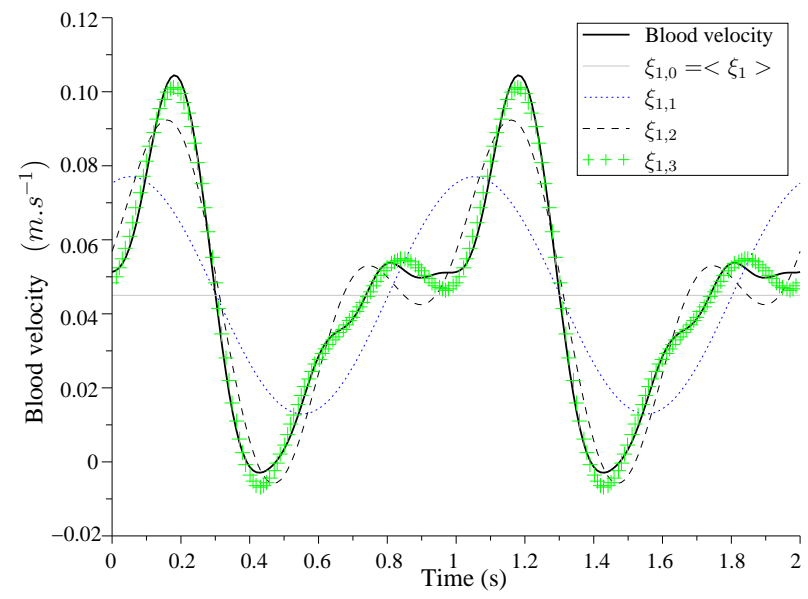

Fig. 2. Blood velocity $\xi_{1}$ (black solid line), mean value $\xi_{1,0}$ (gray solid line) and $n$th order truncated Fourier series $\xi_{1, n}: \xi_{1,1}$ (blue dots), $\xi_{1,2}$ (black dashes) and $\xi_{1,3}$ (green crosses).

estimation and tracking.

This paper addresses the Lyapunov stability of the proposed observer-based controller despite parametric uncertainties. Section II briefly recaps the modeling of an endovascular robot facing a pulsatile blood flow. The synthesis of the adaptive MVT observer-based backstepping controller in then detailed in Section III. This result is illustrated in Section IV by a simulation that shows the robustness to both parametric uncertainties and noise measurement. Conclusions and discussions on open issues are summarized in Section V. 


\section{MOdELING}

Let us consider a microrobot of mass $m$, made of ferromagnetic particles and a payload (ligand, drugs, micelle...), navigating in the arterial network. Any system immersed in a moving fluid is -at least- subjected to two forces: the drag force and the buoyancy force. This section briefly introduces these forces and the magnetic force that controls the robot along a reference trajectory (see e.g. [12] for more details). For sake of simplicity, we here only consider a $1 D$ model.

\section{A. Forces}

1) Hydrodynamic drag force: In a fluidic environment, any system faces the drag force which opposes its motion:

$$
\left\{\begin{aligned}
\vec{F}_{d} & =-\frac{1}{2} \rho_{f} A C_{d}\left(\frac{\|\vec{v}\|}{\beta}\right)^{2} \frac{\vec{v}}{\|\vec{v}\|} \\
C_{d} & =\frac{24}{R e}+\frac{6}{1+\sqrt{R e}}+0.4 \\
R e & =\frac{2 r \rho_{f}\|\vec{v}\|}{\beta \eta}
\end{aligned}\right.
$$

where $A$ is the frontal area, the drag coefficient $C_{d}$ is given by [18], $\beta$ is a dimensionless ratio related to the wall effect caused by the partial vessel occlusion by the robot [19], and $v$ denotes the relative velocity between the robot and the fluid. $\eta$ and $\rho_{f}$ denote respectively the blood viscosity and density. In the case of a spherical microrobot of radius $r$, the drag force can be rewritten as:

$$
\vec{F}_{d}=-\left(a|v|+b v^{2}+c \frac{v^{2}}{1+d \sqrt{|v|}}\right) \frac{\vec{v}}{\|\vec{v}\|}
$$

The parameters in (2) are inherited from (1):

$$
\left\{\begin{aligned}
a & =\frac{6 \pi \eta r}{\beta}, \quad b=\frac{0.2 \rho_{f} \pi r^{2}}{\beta^{2}} \\
c & =\frac{3 \rho_{f} \pi r^{2}}{\beta^{2}}, \quad d=\sqrt{\frac{2 r \rho_{f}}{\beta \eta}}
\end{aligned}\right.
$$

Arterial pulsatile flow profiles are usually modeled using the Womersley model [20], which results in a truncated Fourier series as shown on Fig. 2. Blood velocity $\xi_{1}$ expressed as a $n$th order truncated Fourier series is solution of the $(2 n)$-dimensional autonomous system:

$$
\left(\mathcal{S}_{\xi}\right)\left\{\begin{array}{c}
\dot{\xi}_{1}=\xi_{2} \\
\dot{\xi}_{2}=-\omega^{2}\left(\xi_{1}-\xi_{3}\right) \\
\vdots \\
\dot{\xi}_{2 k-1}=\xi_{2 k} \\
\dot{\xi}_{2 k}=-\omega^{2}\left(k^{2} \xi_{2 k-1}-\xi_{2 k+1}\right) \\
\vdots \\
\dot{\xi}_{2 n}=-\omega^{2} n^{2} \xi_{2 n-1}+\omega^{2} n^{2} v_{m}
\end{array}\right.
$$

with mean blood velocity $v_{m}$. See [16] for technical details.
2) Magnetic force: Three main propulsion designs for magnetic microrobots have been developped: bead pulled, elastic flagellated, and helical tailed robots, see [1] for a survey. In $1 \mathrm{D}$, their magnetic motive force $\vec{F}_{m}$ can be written as [12]:

$$
\vec{F}_{m}=\beta_{2} \vec{u}-\beta_{3} \vec{v}
$$

where $\beta_{3}$ is related to the drag exerting on the helical tail, and is null for elastic flagellated and bead pulled robots. The control input $u$ is the magnetic field gradient $\nabla B$ and the frequency of the oscillating magnetic field $B$ for bead pulling and flagellated robots, respectively. $\beta_{2}$ is proportional to the robot magnetization, radius, and ferromagnetic ratio, denoted $\vec{M}, r$, and $\tau_{m}$ respectively.

3) Apparent weight: The apparent weight of the robot results from the contribution of the weight and the buoyancy:

$$
\vec{W}_{a}=V\left(\rho-\rho_{f}\right) \vec{g}
$$

where the robot density is $\rho=\tau_{m} \rho_{m}+\left(1-\tau_{m}\right) \rho_{p}$ with $\rho_{m}$ and $\rho_{p}$ the magnetic and payload densities, respectively. $V$ denotes the robot volume.

\section{B. State Space Representation}

The reduced system $\left(\mathcal{S}_{r}\right)$ is derived from (2), (5) and (6):

$$
\left(\mathcal{S}_{r}\right)\left\{\begin{aligned}
\dot{z} & =\left[\begin{array}{ll}
0 & 1 \\
0 & 0
\end{array}\right] z+\left[\begin{array}{c}
0 \\
\beta_{2}
\end{array}\right] u+\left[\begin{array}{c}
0 \\
\phi(z, \xi)
\end{array}\right]+\left[\begin{array}{l}
0 \\
1
\end{array}\right] \theta \\
y & =C_{1} z
\end{aligned}\right.
$$

where the state $z$ components $z_{1}$ and $z_{2}$ denote respectively the robot position and velocity, $u$ the control input and $\xi_{1}$ the blood velocity. The output $y$ is the robot position measured by an imager, i.e. $C_{1}=\left[\begin{array}{ll}1 & 0\end{array}\right]$. The function $\phi$ is given by:

$$
\begin{aligned}
\phi(z, \xi)= & {\left[-\sigma\left(z_{2}-\xi_{1}\right)\left(\left(a+\beta_{3}\right)\left|z_{2}-\xi_{1}\right|+b\left(z_{2}-\xi_{1}\right)^{2}\right.\right.} \\
& \left.\left.+c \frac{\left(z_{2}-\xi_{1}\right)^{2}}{1+d \sqrt{\left|z_{2}-\xi_{1}\right|}}\right)\right] \frac{1}{m}
\end{aligned}
$$

where $\theta=V\left(\rho_{f}-\rho\right) g / m$ and $\sigma$ respectively denote the unknown parameter and the sign function.

Let $x=\left[z^{T}, \xi^{T}\right]^{T} \in \mathbb{R}^{2 n+2}$ denote an extended state vector. The extended system is inherited from (7) and (4):

$$
\left(\mathcal{S}_{e}\right):\left\{\begin{aligned}
\dot{x} & =A(0) x+B f(x, u)+B \theta+V_{m} \\
y & =C_{2} x
\end{aligned}\right.
$$

with $B^{T}=\left[\begin{array}{lll}0 & 1 & 0_{1 \times 2 n}\end{array}\right], C_{2}=\left[\begin{array}{ll}C_{1} & 0_{1 \times 2 n}\end{array}\right]$ and $V_{m}^{T}=$ $\left[0_{1 \times(2 n+1)} \omega^{2} n^{2} v_{m}\right]$. Function $f$ is chosen so that $A(0)$ contains the linear part of $\left(\mathcal{S}_{e}\right)$ :

$$
\begin{array}{r}
f(x, u)=\phi(x)+\beta_{2} u+\frac{\left(a+\beta_{3}\right)\left(x_{2}-x_{3}\right)}{m} \\
A(\alpha)=\left[\begin{array}{ccccc}
\chi_{1} & \chi_{2} & O_{2} & \ldots & O_{2} \\
O_{2} & \digamma_{1} & \chi_{3} & \ldots & O_{2} \\
\vdots & \ddots & \ddots & \ddots & \vdots \\
O_{2} & \cdots & \ddots & \digamma_{n-1} & \chi_{3} \\
O_{2} & \cdots & \cdots & O_{2} & \digamma_{n}
\end{array}\right]
\end{array}
$$


with

$$
\begin{array}{lll}
\chi_{1}=\left[\begin{array}{cc}
0 & 1 \\
0 & -\left(\beta_{3}+a\right) / m-\alpha
\end{array}\right] & \chi_{3}=\left[\begin{array}{cc}
0 & 0 \\
\omega^{2} & 0
\end{array}\right] \\
\chi_{2}=\left[\begin{array}{cc}
0 & 0 \\
\left(\beta_{3}+a\right) / m+\alpha & 0
\end{array}\right] & \digamma_{i}=\left[\begin{array}{cc}
0 & 1 \\
-i^{2} \omega^{2} & 0
\end{array}\right]
\end{array}
$$

The knowledge of $x_{1}, x_{2}, x_{3}$ is required to implement a stabilizing control law. Since only $x_{1}$ is measured, an observer is thus necessary. The next section is devoted to the synthesis of the observer-based controller.

\section{MAIN RESULT}

Lemma 1 Let $z_{\text {ref }}=\left[z_{r}(t), \dot{z}_{r}(t), \ddot{z}_{r}(t)\right]$ denote any continuous and bounded reference trajectory, and $\mathcal{K}_{z}$ denote any compact subset of a neighborhood of $\left(z_{r}, \dot{z}_{r}\right) . \forall x \in$ $\mathcal{K}=\mathcal{K}_{z} \times \mathcal{K}_{\xi}$, the systems (7) and (9) satisfies the following properties:

P1) $\phi$ is locally $\gamma$-Lipschitz on $\mathcal{K}$;

P2) System (7) is locally controllable along $z_{\text {ref }}$;

P3) System (9) satisfies $\left(\bar{A}_{1}, \bar{C}\right)$ and $\left(\bar{A}_{2}, \bar{C}\right)$ observable, with $\bar{A}_{1}=\bar{A}(0)$ and $\bar{A}_{2}=\bar{A}\left(\gamma-\frac{a+\beta_{3}}{m}\right)$ with $\bar{A}(\alpha)$ and $\bar{C}$ defined as:

$$
\begin{cases}\bar{A}(\alpha) & =\left[\begin{array}{ll}
A(\alpha) & B \\
O_{1 \times(2 n+2)} & 0
\end{array}\right] \\
\bar{C} & =\left[\begin{array}{ll}
C_{2} & 0
\end{array}\right]\end{cases}
$$

P4) $f$ is differentiable with respect to $x$ and $\forall j \leq 2 n+2$, $\exists\left(a_{j}, b_{j}\right) \in \mathbb{R}^{2}$ such that:

$$
a_{j} \leq \frac{\partial f}{\partial x_{j}}(x, u) \leq b_{j} \quad \forall x \in \mathcal{K}, u \in \mathcal{U}
$$

Proof: Properties (P1) and (P2) have been demonstrated in [16] and [21], respectively. In particular, the local controllability of system (7) is inherited from the controllability of its linearized time-variant system along the reference trajectory $z_{\text {ref }}(t)$ [22], [23].

Let $\Delta$ denote the observability matrix determinant associated with the pair $(\bar{C}, \bar{A}(\alpha))$. It is straightforward that:

$$
\Delta=\left(\left(\beta_{3}+a\right) / m+\alpha\right)^{2 n} \omega^{2 n} \prod_{i=1}^{n} i^{2} \omega^{4(i-1)}
$$

Property (P4) is inherited from (10), from (P1) and from the properties of $\phi$ established in Lemma 1 of [16], namely $\frac{\partial \phi}{\partial x_{2}}=-\frac{\partial \phi}{\partial x_{3}}$ and $\forall x \in \mathcal{K}:$

$$
\frac{a+\beta_{3}}{m} \leq \frac{\partial \phi}{\partial x_{3}}(x) \leq \gamma
$$

Using (10) and (16), it is straightforward that:

$$
\begin{gathered}
0 \leq \frac{\partial f(x, u)}{\partial x_{3}}=-\frac{\partial f(x, u)}{\partial x_{2}} \leq \gamma-\frac{a+\beta_{3}}{m} \\
\frac{\partial f(x, u)}{\partial x_{j}}=0 \text { for all } j=1,4,5, \cdots, 2 n+2
\end{gathered}
$$

\section{A. Observer}

We have previously proposed a high gain observer in [16] to estimate the extended state vector in (9). Yet this observer raises two issues. First, even for small dimensional systems, it is difficult to design the observer in order to obtain relatively low gain $L$ because of the high value of the local Lipschitz constant $\gamma$. Second, even for a blood velocity defined as a first-order truncated Fourier series, the system (9) dimension is 4 . Using higher order truncated series emphasizes this issue since the gain matrix is formed in ascending powers of $L$. That is the reason why we propose another observer based on [24].

Proposition 1 Let $\mathcal{U}$ denote the compact set of admissible inputs. Under assumptions of Lemma (1), $\forall x(0) \in \mathcal{K}$, $\forall \hat{x}(0) \in \mathcal{K}, \forall \hat{\theta}(0) \in \mathcal{K}_{\theta}, \forall u \in \mathcal{U}$ :

$$
\left\{\begin{aligned}
\dot{\hat{x}} & =A(0) \hat{x}+B(f(\hat{x}, u)+\hat{\theta})+K\left(y-C_{2} \hat{x}\right)+V_{m} \\
\hat{y} & =C_{2} \hat{x} \\
\dot{\hat{\theta}} & =K_{\theta}\left(y-C_{2} \hat{x}\right)
\end{aligned}\right.
$$

is an exponential observer of system (9) on $\mathcal{K} \times \mathcal{K}_{\theta}$ with the symmetric positive definite matrices $P_{o}, Q_{o}$ and the gain $\bar{K}=\left(K^{T} K_{\theta}^{T}\right)^{T}$ that satisfy the Linear Matrix Inequalities:

$$
\left(\bar{A}_{i}-\bar{K} \bar{C}\right)^{T} P_{o}+P_{o}\left(\bar{A}_{i}-\bar{K} \bar{C}\right)<-Q_{o}, \forall i \in\{1,2\}
$$

Proof: Let $\tilde{x}=x-\hat{x}$ denote the estimation error. Let $C o(x, \hat{x})=\{\lambda x+(1-\lambda) \hat{x}, 0 \leq \lambda \leq 1\}$ and $\bar{C} o(x, \hat{x})$ denote a convex and its convex hull. Applying the MVT to $f, \exists \varrho(t) \in \bar{C} o(x, \hat{x})$ such that:

$$
\tilde{f}=f(x, u)-f(\hat{x}, u)=\left(\sum_{j=1}^{2 n+2} e_{j}^{T} \frac{\partial f}{\partial x_{j}}(\varrho(t), u(t))\right) \tilde{x}(t)
$$

with $\left\{e_{1}, \ldots e_{2 n+2}\right\}$ denoting the canonical basis of $\mathbb{R}^{2 n+2}$.

$$
\begin{aligned}
& \text { Let } \quad \mathcal{A}(h(t))=A(0)+B \sum_{j=1}^{2 n+2} h_{j}(t) e_{j}^{T} \quad \text { with } \\
& h_{j}(t)=\frac{\partial f}{\partial x_{j}}(\varrho(t), u(t)) .
\end{aligned}
$$

Then, using (9) and (18), the estimation error dynamics satisfies the LPV system:

$$
\left\{\begin{aligned}
\dot{\tilde{x}} & =\left(\mathcal{A}(h(t))-K C_{2}\right) \tilde{x}+B \tilde{\theta} \\
\dot{\tilde{\theta}} & =-K_{\theta}\left(y-C_{2} \hat{x}\right)
\end{aligned}\right.
$$

where $\tilde{\theta}=\theta-\hat{\theta}$ denotes the parameter estimation error.

Let $\epsilon=\left(\tilde{x}^{T}, \tilde{\theta}\right)^{T}$, then system (21) can be rewritten as

$$
\dot{\epsilon}=(\overline{\mathcal{A}}(h(t))-\bar{K} \bar{C}) \epsilon
$$

with

$$
\overline{\mathcal{A}}(h(t))=\left[\begin{array}{ll}
\mathcal{A}(h(t)) & B \\
O_{1 \times(2 n+2)} & 0
\end{array}\right]
$$


Property (P4) implies that the parameter $h(t)$ evolves in a bounded set $\mathcal{H}$ whose vertex $\mathcal{V}$ is given by:

$$
\mathcal{V}=\left\{\alpha=\left(\alpha_{1}, \cdots, \alpha_{2 n+2}\right) \mid \alpha_{j} \in\left\{a_{j}, b_{j}\right\}\right\}
$$

From $(\mathbf{P} 4), \mathcal{V}$ reduces to $\mathcal{V}=\left\{a_{3}, b_{3}\right\}=\left\{0, \gamma-\frac{a+\beta_{3}}{m}\right\}$.

For all $P_{o}$ symmetric positive definite, a candidate Lyapunov function is given by:

$$
V_{o}(\epsilon)=\epsilon^{T} P_{o} \epsilon \leq \bar{\lambda}\|\epsilon\|^{2}
$$

with $\bar{\lambda}$ the highest eigenvalue of $P_{o}$.

Differentiating (25) using (22) leads to:

$$
\dot{V}_{o}(\epsilon)=\epsilon^{T} Q(h(t)) \epsilon
$$

with the time-varying matrix $Q(h)$ affine in $h$ defined by:

$$
Q(h(t))=(\overline{\mathcal{A}}(h(t))-\bar{K} \bar{C})^{T} P_{o}+P_{o}(\overline{\mathcal{A}}(h(t))-\bar{K} \bar{C})
$$

Let $\bar{K}, P_{o}$ and $Q_{o}$ satisfy (19). Since $\bar{A}\left(a_{3}\right)=\bar{A}_{1}$ and $\bar{A}\left(b_{3}\right)=\bar{A}_{2}$, we consequently have $Q(\alpha)<-Q_{o}$ $\forall \alpha \in \mathcal{V}$. Using the principle of convexity, we then have $Q(h(t))<-Q_{o}, \forall h \in \mathcal{H}$.

Hence, we have

$$
\dot{V}_{o}(\epsilon)<-\epsilon^{T} Q_{o} \epsilon
$$

Let $\underline{\lambda}$ denote the smallest eigenvalue of $Q_{o}$, we then have

$$
\dot{V}_{o}(\epsilon)<-\underline{\lambda}\|\epsilon\|^{2} \leq-(\underline{\lambda} / \bar{\lambda}) V_{o}(\epsilon)
$$

It follows from (29) that (18) is an exponential observer for system (9).

\section{B. Controller}

Proposition 2 Under assumptions of Lemma (1), the backstepping control law $u=\kappa(x, \theta)$ :

$\kappa(x, \theta)=-\frac{\left(k_{2}+k_{1}\right) \bar{z}_{2}+\left(1-k_{1}^{2}\right) \bar{z}_{1}+\phi(x)+\theta-\ddot{z}_{r}}{\beta_{2}}$

stabilizes the system (7) along any $\mathcal{C}^{0}$ reference trajectory for any bounded initial state $x(0)$ with the controller gains $k_{1}, k_{2}>0$ and $\bar{z}_{1}=z_{1}-z_{r}, \bar{z}_{2}=z_{2}+k_{1} z_{1}-\dot{z}_{r}$.

Proof: Let

$$
\left\{\begin{array}{l}
\bar{z}_{1}=z_{1}-z_{r} \\
\bar{z}_{2}=z_{2}-\dot{z}_{r}-\alpha
\end{array}\right.
$$

for some stabilizing function $\alpha$.

- A candidate Lyapunov function is:

$$
V_{1}=\frac{1}{2} \bar{z}_{1}^{2} \Longrightarrow \dot{V}_{1}=\bar{z}_{1}\left(z_{2}-\dot{z}_{r}\right)=\bar{z}_{1}\left(\bar{z}_{2}+\alpha\right)
$$

Setting $\alpha=-k_{1} \bar{z}_{1}$ leads to:

$$
\dot{V}_{1}=-k_{1} \bar{z}_{1}^{2}+\bar{z}_{1} \bar{z}_{2}
$$

Since $\dot{\bar{z}}_{1}=z_{2}-\dot{z}_{r}=\bar{z}_{2}+\alpha=\bar{z}_{2}-k_{1} \bar{z}_{1}$, we obtain:

$$
\dot{\bar{z}}_{2}=\phi(x)+\theta+\beta_{2} u+k_{1}\left(\bar{z}_{2}-k_{1} \bar{z}_{1}\right)-\ddot{z}_{r}
$$

- In this last step, the candidate Lyapunov function is:

$$
V_{2}=V_{1}+\frac{1}{2} \bar{z}_{2}^{2}
$$

Differentiating (35) using (34), we obtain:

$$
\dot{V}_{2}=-k_{1} \bar{z}_{1}^{2}+\bar{z}_{2}\left(\phi(x)+\theta+\beta_{2} u+k_{1} \bar{z}_{2}+\left(1-k_{1}^{2}\right) \bar{z}_{1}-\ddot{z}_{r}\right)
$$

Using $u=\kappa(x, \theta)$ given by (30) leads to:

$$
\dot{V}_{2}=-k_{1} \bar{z}_{1}^{2}-k_{2} \bar{z}_{2}^{2}
$$

\section{Stability of the observer-based controller}

Proposition 3 Under assumptions of Proposition (1), the observer-based control law $\hat{u}=\kappa(\hat{x}, \hat{\theta})$ :

$\kappa(\hat{x}, \hat{\theta})=-\frac{\left(k_{2}+k_{1}\right) \hat{\bar{z}}_{2}+\left(1-k_{1}^{2}\right) \hat{\bar{z}}_{1}+\phi(\hat{x})+\hat{\theta}-\ddot{z}_{r}}{\beta_{2}}$

stabilizes the system (7) along any $\mathcal{C}^{0}$ reference trajectory for any initial state $(x(0), \hat{x}(0)) \in \mathcal{K}^{2}$ and $\hat{\theta}(0) \in \mathcal{K}_{\theta}$ with the controller gains $k_{1}, k_{2}>0, \hat{\bar{z}}_{1}=\hat{z}_{1}-z_{r}, \hat{\bar{z}}_{2}=\hat{z}_{2}+k_{1} \hat{z}_{1}-\dot{z}_{r}$ and $\tilde{z}=z-\hat{z}$. obtain:

Proof: Replacing $u$ with $\hat{u}$ in the equation (36), we

$$
\begin{aligned}
\dot{V}_{2} & =-k_{1} \bar{z}_{1}^{2}+\bar{z}_{2}\left(\tilde{\phi}+\left(k_{1}+k_{2}\right) \tilde{\bar{z}}_{2}+\left(1-k_{1}^{2}\right) \tilde{\bar{z}}_{1}-k_{2} \bar{z}_{2}+\tilde{\theta}\right) \\
& =-k_{1} \bar{z}_{1}^{2}-k_{2} \bar{z}_{2}^{2}+\bar{z}_{2}\left(\tilde{\phi}+\left(k_{1}+k_{2}\right) \tilde{\bar{z}}_{2}+\left(1-k_{1}^{2}\right) \tilde{\bar{z}}_{1}+\tilde{\theta}\right)
\end{aligned}
$$

with $\tilde{\phi}=\phi(x)-\phi(\hat{x})$. Using property (P1) leads to:

$$
\begin{aligned}
\dot{V}_{2} & \leq-k_{1}\left\|\bar{z}_{1}\right\|^{2}-k_{2}\left\|\bar{z}_{2}\right\|^{2}+\gamma\left\|\bar{z}_{2}\right\|\|\tilde{x}\| \\
& +\left(k_{1}+k_{2}\right)\left\|\bar{z}_{2}\right\|\left\|\tilde{\bar{z}}_{2}\right\|+\left(1-k_{1}^{2}\right)\left\|\bar{z}_{2}\right\|\left\|\tilde{\bar{z}}_{1}\right\|+\left\|\bar{z}_{2}\right\|\|\tilde{\theta}\|
\end{aligned}
$$

Yet we have the following bound:

$$
\begin{aligned}
\bar{z}_{2}=z_{2}-\dot{z}_{r}+k_{1} \bar{z}_{1} & \Longrightarrow \tilde{\bar{z}}_{2}=\tilde{z}_{2}+k_{1} \tilde{\bar{z}}_{1} \\
& \Longrightarrow\left\|\tilde{\bar{z}}_{2}\right\| \leq\left\|\tilde{z}_{2}\right\|+k_{1}\left\|\tilde{\bar{z}}_{1}\right\|
\end{aligned}
$$

So using (41) in (40) yields:

$$
\begin{aligned}
\dot{V}_{2} & \leq-k_{1}\left\|\bar{z}_{1}\right\|^{2}-k_{2}\left\|\bar{z}_{2}\right\|^{2}+\gamma\left\|\bar{z}_{2}\right\|\|\tilde{x}\| \\
& +\left(k_{1}+k_{2}\right)\left\|\bar{z}_{2}\right\|\left\|\tilde{z}_{2}\right\|+\left(1+k_{1} k_{2}\right)\left\|\bar{z}_{2}\right\|\left\|\tilde{\bar{z}}_{1}\right\|+\left\|\bar{z}_{2}\right\|\|\tilde{\theta}\| \\
& \leq-k_{1}\left\|\bar{z}_{1}\right\|^{2}-k_{2}\left\|\bar{z}_{2}\right\|^{2}+\gamma\left\|\bar{z}_{2}\right\|\|\tilde{x}\| \\
& +\left(1+k_{1} k_{2}+k_{1}+k_{2}\right)\left\|\bar{z}_{2}\right\|\|\tilde{x}\|+\left\|\bar{z}_{2}\right\|\|\tilde{\theta}\| \\
& \leq-\eta_{c}\|\bar{z}\|^{2}+\zeta_{c}\|\bar{z}\|\|\epsilon\|
\end{aligned}
$$

with $\eta_{c}=\min \left(k_{1}, k_{2}\right)$ and $\zeta_{c}=\left(2+\gamma+k_{1}+k_{2}+k_{1} k_{2}\right)$.

Consider the Lyapunov function $W(\bar{z}, \epsilon)$ with $\zeta>0$ :

$$
W(\bar{z}, \epsilon)=\zeta V_{2}(\bar{z})+V_{o}(\epsilon)
$$

Using (42) and (29), the time derivative of $W(\bar{z}, \epsilon)$ is given by:

$$
\dot{W}(\bar{z}, \epsilon) \leq-\zeta \eta_{c}\|\bar{z}\|^{2}+\zeta \zeta_{c}\|\bar{z}\|\|\epsilon\|-\underline{\lambda}\|\epsilon\|^{2}
$$

Choosing $\zeta=\eta_{c} \underline{\lambda} / \zeta_{c}^{2}$ thus results in:

$$
\dot{W}(\bar{z}, \epsilon) \leq-\zeta \eta_{c}\|\bar{z}\|^{2} / 2-\underline{\lambda}\|\epsilon\|^{2} / 2
$$


TABLE I

PARAMETERS NOMINAL VALUES

\begin{tabular}{|c||c|c|}
\hline Blood viscosity & $\eta$ & $16 \times 10^{-3}[\mathrm{~Pa} . \mathrm{s}]$ \\
\hline Blood density & $\rho_{f}$ & $1060\left[\mathrm{~kg} \cdot \mathrm{m}^{-3}\right]$ \\
\hline Ferromagnetic density & $\rho_{m}$ & $7500\left[\mathrm{~kg} \cdot \mathrm{m}^{-3}\right]$ \\
\hline Robot radius & $r$ & $2.510^{-4}[\mathrm{~m}]$ \\
\hline Vessel diameter & $D$ & $310^{-3}[\mathrm{~m}]$ \\
\hline Payload density & $\rho_{p}$ & $1500\left[\mathrm{~kg} \cdot \mathrm{m}^{-3}\right]$ \\
\hline Ferromagnetic ratio & $\tau_{m}$ & 0.75 \\
\hline Magnetization & $M$ & $1.23 \times 10^{6}\left[\mathrm{A.m} \mathrm{m}^{-1}\right]$ \\
\hline Controller gains & $\left(k_{1}, k_{2}\right)$ & $(7,14)$ \\
\hline Mean blood velocity & $v_{m}$ & $0.05\left[{\left.\mathrm{~m} . \mathrm{s}^{-1}\right]}\right.$ \\
\hline
\end{tabular}

TABLE II

INITIAL CONDITIONS FOR THE SYSTEM AND THE OBSERVER

\begin{tabular}{|c|c|}
\hline$\left(x^{T}(0) \theta\right)$ & $\left(\begin{array}{llllll}0 & 0 & 0.1075 & 0 & -8.179\end{array}\right)$ \\
\hline$\left(\hat{x}^{T}(0) \hat{\theta}(0)\right)$ & $\left(\begin{array}{lllll}0.001 & 0.01 & 0 & 0.001 & -9\end{array}\right)$ \\
\hline
\end{tabular}

Set $\kappa_{1}=\max (\zeta / 2, \bar{\lambda})$ and $\kappa_{2}=\min \left(\zeta \eta_{c}, \underline{\lambda}\right) / 2$, then we can write:

$$
\begin{aligned}
& W(\bar{z}, \epsilon) \leq \kappa_{1}\left(\|\bar{z}\|^{2}+\|\epsilon\|^{2}\right) \\
& \dot{W}(\bar{z}, \epsilon) \leq-\kappa_{2}\left(\|\bar{z}\|^{2}+\|\epsilon\|^{2}\right)
\end{aligned}
$$

We consequently have:

$$
\dot{W}(\bar{z}, \epsilon) \leq-\frac{\kappa_{2}}{\kappa_{1}} W(\bar{z}, \epsilon)
$$

Therefore $\bar{z}$ and $\epsilon=\left(\tilde{x}^{T}, \tilde{\theta}\right)^{T}$ exponentially converge to zero, and the same goes for $z, \tilde{x}$ and $\tilde{\theta}$.

\section{Simulation Results}

The simulation is performed by taking into account the limitations of the actuators. In order to not exceed the capacity of the magnetic device, the control inputs are timescaled as $u_{a}(t)=u(t) / k(t)$ with $k(t)=\max \left[1,|u(t)| / u_{\text {sat }}\right]$. The microrobot position is assumed to be measured within an accuracy of $100 \mu \mathrm{m}$ consistent with the best imagers resolution. A Gaussian white noise with a standard deviation of $100 \mu \mathrm{m}$ is thus added to the measured output. The nominal values of parameters are given in Table I. The robot is bead pulled, so that we have $\beta_{2}=\frac{\tau_{m} M}{\rho}$ and $\beta_{3}=0$. Without loss of generality, the reference trajectory is a fixed point. The blood velocity is modeled by a first-order Fourier series. Table II gives the initial conditions for the system and the observer.

The MVT observer gain is:

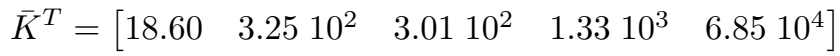

The simulation shows that the MVT-observer-based controller is stable despite the presence of measurement noise. The Figure 3 (resp. 4) illustrates the real and estimated microrobot positions (resp. velocities) and the associated estimation errors. The latter have converged in less than 1.5s. The microrobot position and velocity converges to zero after a $2 s$ long transient phase that corresponds to the

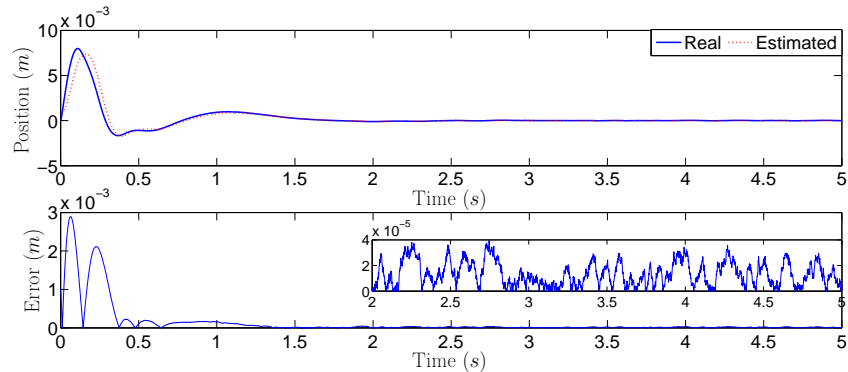

Fig. 3. Real and estimated positions / Position estimation error.

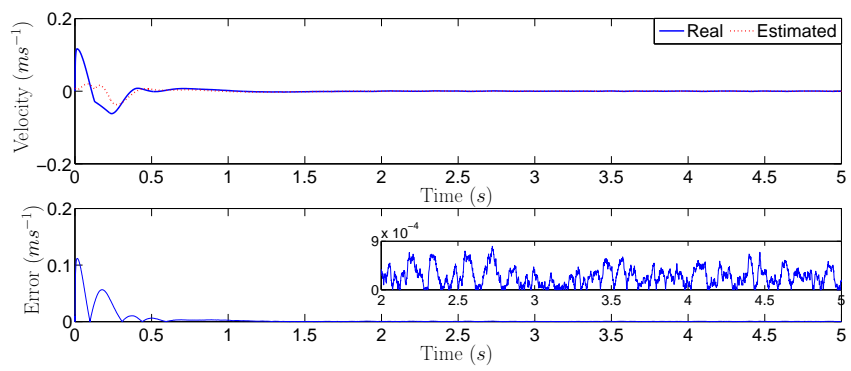

Fig. 4. Real and estimated velocities / Velocity estimation error.

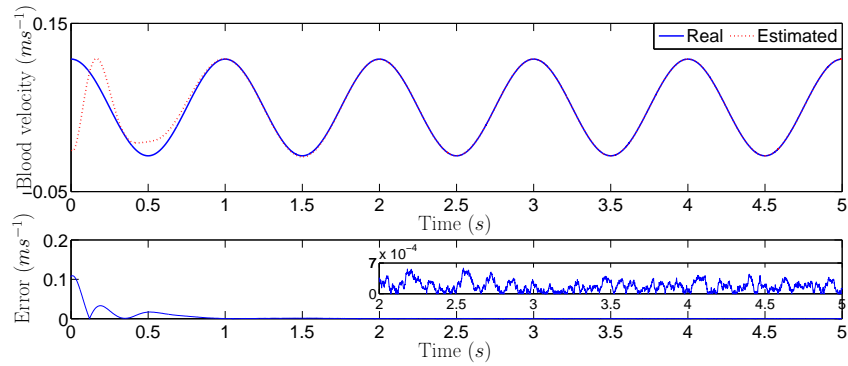

Fig. 5. Real and estimated Blood velocities / Blood velocity estimation error.

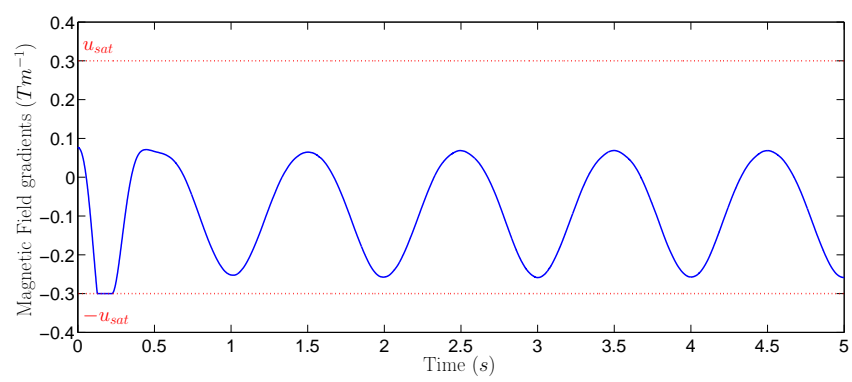

Fig. 6. Control input: $\nabla B$.

parametric error convergence time. The tracking is degraded at the beginning of the simulation because the control input reaches the saturation. Once stabilized the position and velocity estimation errors are less than $40 \mu \mathrm{m}$ and $900 \mu \mathrm{m} . \mathrm{s}^{-1}$, respectively. Similarly, the Figure 5 shows that the blood velocity estimation error is less than $700 \mu \mathrm{m} . \mathrm{s}^{-1}$ once the observer has converged. The Figure 6 illustrates that the control input is not too much affected by noise. The Figure 7 illustrates the real and estimated parameter and the associated estimation error: convergence occurs within $2 s$. 

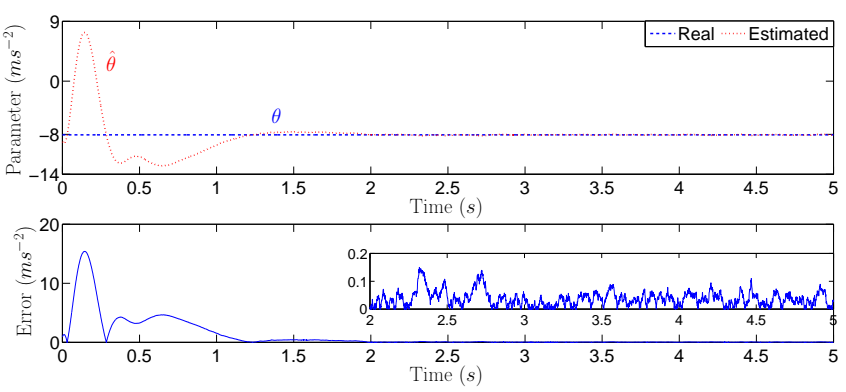

Fig. 7. On-line estimation of the unknown parameter and parametric error $\tilde{\theta}$.

\section{DisCUSSION AND CONCLUSION}

Many studies on the measurement of the average blood velocity are available in the literature. Unfortunately, this information is neither sufficient nor accurate enough for controlling microrobots navigating in the vasculature. The drag force has a major impact on the dynamic behavior of the system and this force strongly and nonlinearly depends on the relative velocity between the robot and the time-varying blood flow. The blood velocity is pulsatile and periodic. Since any periodic signal has a Fourier series decomposition, it is then possible to define a dynamic model of the blood velocity, yielding to an extending system.

We have proposed an observer-based controller using the sole measurement of the microrobot position obtained thanks to an imager. Since the nonlinear modeling describes both physical and physiological forces, it is also affected by many biological parameters uncertainties. To robustify the tracking with respect to these uncertainties, an adaptive observer-based controller has been developed and its relevancy and efficiency is illustrated through simulation. The parametric uncertainty acts on the dynamics of non measurable state, which is a difficult case to deal with. Yet the system is stabilized despite noise measurement.

Extension of the approach to a $2 \mathrm{D}$ model is an ongoing work. The main difficulty lies in the necessity to take into account the electrostatic force, which may raise some issues about the Lipschitz constant which in turn induces high gains and sensitivity to noise. The estimation of the blood pulsation is still an open issue, since the resulting perturbation model becomes nonlinear and non uniformly observable.

\section{REFERENCES}

[1] B. J. Nelson, I. K. Kaliakatsos, and J. J. Abbott, "Microrobots for minimally invasive medecine," Annual Review of Biomedical Engineering, vol. 12, pp. 55-85, 2010.

[2] G. Kosa, M. Shoham, and M. Zaaroor, "Propulsion method for swimming microrobots," IEEE Transactions on Robotics, vol. 23(1), pp. 137-150, 2007.

[3] L. Zhang, J. J. Abbott, L. X. Dong, B. E. Kratochvil, D. J. Bell, and B. J. Nelson, "Artificial bacterial flagella: Fabrication and magnetic control," Applied Physics Letters, vol. 94(6), 2009.
[4] M. C. Lagomarsino, F. Capuani, and C. P. Lowe, "A simulation study of the dynamics of a driven filament in an Aristotelian fluid," Journal of Theoretical Biology, vol. 224(2), pp. 215-224, 2003.

[5] A. A. Evans and E. Lauga, "Propulsion by passive filaments and active flagella near boundaries," Physical Review E, vol. 82(4), pp. 041 9151-041915-12, 2010

[6] R. Dreyfus, J. Beaudry, M. L. Roper, M. Fermigier, H. A. Stone, and J. Bibette, "Microscopic artificial swimmers," Nature, vol. 437, pp. 862-865, 2005.

[7] J. Edd, S. Payen, B. Rubinsky, M. L. Stoller, and M. Sitti, "Biomimetic propulsion for a swimming surgical microrobot," IEEE International Conference on Intelligent Robots and Systems, Las Vegas, pp. 2583$2588,2003$.

[8] L. Zhang, K. E. Peyer, and B. J. Nelson, "Artificial bacterial flagella for micromanipulation," Lab on a chip, vol. 10, no. 17, pp. 2203-2215, 2010.

[9] J. J. Abbott, K. E. Peyer, M. C. Lagomarsino, L. Zhang, L. X. Dong, I. K. Kaliakatsos, and B. J. Nelson, "How should microrobots swim?" International Journal of Robotics Research, vol. 28, pp. 1434-1447, 2009.

[10] J.-B. Mathieu, G. Beaudoin, and S. Martel, "Method of propulsion of a ferromagnetic core in the cardiovascular system through magnetic gradients generated by an MRI system," IEEE Transactions on Biomedical Engineering, vol. 53, no. 2, pp. 292-299, 2006.

[11] S. Martel and M. Mohammadi, "Using a swarm of self-propelled natural microrobots in the form of flagellated bacteria to perform complex micro-assembly tasks," IEEE International Conference on Robotics and Automatics, pp. 500-505, 2010.

[12] L. Arcese, M. Fruchard, and A. Ferreira, "Endovascular magneticallyguided robots: navigation modeling and optimization," IEEE Transactions on Biomedical Engineering, vol. 59(4), pp. 977-987, 2012.

[13] P. Vartholomeos and C. Mavroidis, "In silico studies of magnetic microparticle aggregations in fluid environments for MRI-guided drug delivery," IEEE Transactions on Biomedical Engineering, vol. 59(11), pp. 3028-3038, 2012.

[14] R. Ponzini, C. Vergara, G. Rizzo, A. Veneziani, A. Roghi, A. Vanzulli, O. Parodi, and A. Redaelli, "Womersley number-based estimates of blood flow rate in doppler analysis: In vivo validation by means of phase-contrast MRI," IEEE Transactions on Biomedical Engineering, vol. 57(7), pp. 1807-1815, 2010.

[15] A. Zemouche and M. Boutayeb, "A unified $H_{\infty}$ adaptive observer synthesis method for a class of systems with both lipschitz and monotone nonlinearities," Systems \& Control Letters, vol. 58, no. 4, pp. $282-288,2009$.

[16] M. Fruchard, L. Arcese, and E. Courtial, "Estimation of the blood velocity for nanorobotics," IEEE Transactions on Robotics, vol. 30, no. 1, pp. 93-102, 2014.

[17] L. Sadelli, M. Fruchard, and A. Ferreira, "Observer-based controller for microrobot in pulsatile blood flow," IEEE International Conference on Decision and Control, submitted for publication.

[18] F. White, Viscous Fluid Flow. McGraw Hill New-York, 1991.

[19] R. Kehlenbeck and R. D. Felice, "Empirical relationships for the terminal settling velocity of spheres in cylindrical columns," Chemical Eng. Technology, vol. 21, pp. 303-308, 1999.

[20] J. Womersley, "Method for the calculation of velocity, rate of flow and viscous drag in arteries when the pressure gradient is known," The Journal of physiology, vol. 127(3), pp. 553-563, 1955.

[21] L. Arcese, M. Fruchard, and A. Ferreira, "Adaptive controller and observer for a magnetic microrobot," IEEE Transactions on Robotics, vol. 29(4), pp. 1060-1067, 2013.

[22] L. M. Silverman and H. E. Meadows, "Controllability and observability in time-variable linear systems," SIAM Journal on Control and Optimization, vol. 5, pp. 64-73, 1967.

[23] A. Isidori, Nonlinear control systems. Springer-Verlag London, 1995.

[24] A. Zemouche and M. Boutayeb, "On Imi conditions to design observers for lipschitz nonlinear systems," Automatica, vol. 49, no. 2, pp. $585-591,2013$. 